\title{
Reuniones de morbilidad y mortalidad, ¿una estrategia de aprendizaje?
}

\author{
Catalina Castañeda-Motta ${ }^{1}$, Neil Valentín Vega-Peña ${ }^{2}$ \\ Si al final todo resulta bien, entonces lo que se diga en mi contra no importará. \\ Si me equivoco, diez ángeles jurando que tenía razón no harán la diferencia. \\ Abraham Lincoln
}

\section{RESUMEN}

Las reuniones o conferencias de morbilidad y mortalidad (M\&M), una práctica común en todo el mundo, buscan evaluar los eventos adversos y las complicaciones relacionadas con la atención médica, desde una perspectiva académica y considerando un mejoramiento en la calidad de la atención hospitalaria. Sin embarơo, en nuestro país, su ejecución es un desafío metodológico debido a la dificultad para su conceptualización, implementación, evaluación de su efectividad y utilidad como herramienta educativa; al estar carente de una estructura formalizada que considere estos temas.

El objetivo de este trabajo es evaluar su papel desde una perspectiva académica individual e institucional, considerando diferentes teorías y modelos que podrían apoyar el M\&M (teoría del aprenđizaje experimental, teoría de la actividad en el aprenđizaje sociocultural, cultura justa, teoría de la seguridad del modelo y teoría de la segunda víctima), con el fin de responder la pregunta: ¿son las reuniones de M\&M una estrategia de aprendizaje?

\section{PALABRAS CLAVE}

Aprendizaje; Educación Médica; Métodos; Morbilidad; Mortalidad

\footnotetext{
1 Residente IV año Cirugía General. Universidad de la Sabana. Facultad de Medicina. Departamento de Cirugía, Bogotá.

2 Profesor Asistente Universidad de la Sabana. Facultad de Medicina. Departamento de Cirugía, Bogotá.

Correspondencia: Neil Valentín Vega-Peña; neil.valentin@unisabana.edu.co

Recibido: agosto 8 de 2019

Aceptado: noviembre 12 de 2019
}

Cómo citar: Castañeda-Motta C, Vega-Peña NV. Reuniones de morbilidad y mortalidad, iuna estrategia de aprendizaje? latreia. 2020 Jul-Sep;33(3):286-97. D0I 10.17533/udea. iatreia.52. 


\section{SUMMARY}

\section{Morbidity and mortality meetings or conferences, a learning strategy?}

Morbidity and mortality (M\&M) meetings or conferences, a common practice worldwide, seek to assess adverse events and complications related to medical care, from an academic perspective and considering an improvement in the quality of hospital care. However, in our country, its execution is a methodological challenge due to the difficulty for its conceptualization, implementation, evaluation of its effectiveness and usefulness as an educational tool, since it lacks a formalized structure that considers these issues.

The aim of this work is to evaluate its role from an individual and institutional academic perspective, considering the different theories and models that could support M\&M (theory of experimental learning, activity theory in sociocultural learning, just culture, security theory model and theory of the second victim), in order to answer the question: are M\&M meetings a learning strategy?

\section{KEYWORDS}

Learning; Medical Education; Methods; Morbidity; Mortality

\section{INTRODUCCIÓN}

Desde la introducción del trabajo del Dr. Ernest A. Codman, cirujano de principios del siglo xx en EE. Uu., las M\&M buscan evaluar los eventos adversos y las complicaciones derivadas de la atención a los pacientes ${ }^{(1)}$, con el propósito de mejorar la calidad de la atención, prevenir al máximo desenlaces desfarorables y generar una estrategia de aprendizaje tanto en los profesionales en entrenamiento como en los ya formados ${ }^{(2)}$.

Actualmente, las M\&M son una práctica común en los departamentos quirúrogicos y clínicos de todo el mundo, siendo obligatoria en los programas de formación de residentes en Ios EE. UU. ${ }^{(3)}$, aun cuando no existe evidencia suficiente que apoye su utilidad, vista desde el impacto favorable en la calidad de la atención y la seguridad del paciente ${ }^{(4-5)}$.

Su alcance, dentro del análisis de eventos adversos y las complicaciones desde una perspectiva organizacional, responde a la ejecución de las políticas institucionales que, en Colombia, dan cumplimiento a un marco normativo estatal establecido en los Lineamientos para la Política de Seguridad del Paciente del Ministerio de la Protección Social, pues deben existir estrategias encaminadas a "prevenir la ocurrencia de situaciones que afecten la seguridad del paciente, reducir y, de ser posible, eliminar la ocurrencia de eventos adversos para contar con instituciones seguras y competitivas internacionalmente" (6).

De acuerdo con la Organización Mundial de la Salud (OMS), 1 de cada 10 pacientes sufren algún tipo de daño durante la estancia hospitalaria, donde hasta un $50 \%$ de ellos son totalmente evitables ${ }^{(7)}$, lo cual pone de manifiesto la magnitud del problema y demanda una intervención prioritaria a todo nivel, incluso durante la formación del personal asistencial a cargo del cuidado de los pacientes.

Como parte de lo anterior, se ha señalado un papel favorable de las M\&M en la educación de los futuros médicos en el pregrado y posgrado, mediante el aprendizaje de los errores propios o ajenos, el análisis de las políticas administrativas de las instituciones y del sistema de salud en la atención médica y las complejas interacciones de los actores involucrados en los procesos asistenciales ${ }^{(8-9)}$. De igual manera, su ejecución constituye un desafío dada la dificultad para su conceptualización e implementación, la evaluación de su efectividad en el logro de los objetivos propuestos y su pertinencia como herramienta educativa en un escenario complejo para los profesionales en entrenamiento (o formados) en las áreas clínicas y quirúrogicas.

Por lo anterior, el objetivo del presente escrito es evaluar el papel de las M\&M desde una perspectiva académica de acuerdo con el soporte teórico de las mismas, buscando responder el interrogante ¿son las M\&M una estrategia de aprendizaje?

Para abordar la pregunta planteamos los siguientes apartados: 1. Aspectos conceptuales y contexto de ejecución, 2. Marco teórico, 3. Análisis de los procesos 
e implementación de las M\&M y, 4. Papel en la educación médica.

El presente documento se referirá a las "reuniones de morbilidad y mortalidad" (morbidity and mortality meetings, en inglés) en lugar de "conferencias de morbilidad y mortalidad" (morbidity and mortality conferences), debido a la posible confusión con las exposiciones magistrales sobre un tema específico en los escenarios académicos hospitalarios también Ilamadas "conferencias".

\section{ASPECTOS CONCEPTUALES Y CONTEXTO DE EJECUCIÓN}

Las M\&M son escenarios de discusión de casos clínicos intrahospitalarios, generalmente, en instituciones académicas donde se analizan los indicadores de resultados de los servicios asistenciales (mortalidad, complicaciones, eventos adversos e incidentes, entre otros), con el fin de identificar posibles focos de intervención ante un eventual error o deficiencia en los procesos de atención del paciente involucrado ${ }^{(2,5)}$.

Este análisis de desenlaces clínicos, no esperados o desfavorables, se considera un elemento evaluativo de la calidad hospitalaria de obligatorio cumplimiento en las instituciones prestadoras de servicios de salud (IPS), independiente de su actividad académica o régimen de atención en salud ${ }^{(10)}$. De acuerdo con el Estudio IBEAS. Prevalencia de eventos adversos en hospitales en Latinoamérica, se documentó una prevalencia de un 13,2\% de eventos adversos en CoIombia ${ }^{(1)}$ que, al ser superior a las cifras reportadas por la OMS para el mundo $(10 \%)^{(7)}$, hace pertinente hoy a las M\&M como una estrategia de impacto ante este problema, según su carácter analítico basado en el aprendizaje y el mejoramiento en la actividad del personal asistencial y las instituciones ${ }^{(3)}$.

La efectividad de una M\&M en sus propósitos organizacionales se logra cuando:

- Permite identificar eventos que resultan en desenlaces adversos para el paciente.

- Promueve la discusión de eventos adversos.

- Identifica y difunde información acerca de las perspectivas sobre la atención al paciente, considerando la evidencia y la experiencia.
- Refuerza la responsabilidad para brindar atención de alta calidad.

- Se crea un escenario en el que los médicos reconocen y discuten las razones de los errores ${ }^{(12)}$.

Cada uno de los anteriores objetivos pretende una mejoría futura en la atención de los pacientes y tiene implícito un impacto positivo en la educación médica del personal involucrado en ella.

En nuestro medio, las M\&M se conocen como "reuniones de complicaciones y mortalidad", "junta de decisiones y complicaciones", "reunión semanal del servicio", "Ateneo", entre otros nombres, de acuerdo con la trađición establecida en la institución respecti$\nabla a{ }^{(13)}$. Estas denominaciones han sido documentadas por los autores luego de la participación en estas reuniones, como parte de su entrenamiento en el pregrado, el postorado y en su ejercicio médico profesional en diferentes centros hospitalarios del país.

Las M\&M son consideradas como el momento propicio para la discusión de los problemas administrativos concurrentes, la socialización de la estadística de procedimientos y la toma de decisiones de casos complejos, generalmente por consenso, involucrándose dentro de la junta médica del servicio o la junta de decisiones del departamento (experiencia de los autores, ya descrita).

Pretender abarcar múltiples temas dentro de un espacio limitado por la complejidad de los casos a analizar, el tiempo de los asistentes y la continuidad de las actividades propias del servicio es un obstáculo para los propósitos académicos y de evaluación de los resultados, por ello, se convierte en una oportunidad para implementar estrategias en el abordaje de los problemas, en un espacio y tiempo limitados.

Las M\&M deben ser concebidas como un escenario integrador al evaluar $y$ gestionar una política de mejoramiento de la calidad hospitalaria mediante un análisis de eventos adversos, generando así, explícitamente, educación médica en todos los niveles (pregrado, posgrado y profesionales en ejercicio), incluso en las IPS con un perfil no académico dentro de su misión y visión institucionales ${ }^{(14)}$. Su ejecución constituye un modelo de aprendizaje que analiza metódicamente los factores que conducen al desenlace negativo y establece una valoración objetiva 
de la seguridad de la atención del paciente en la institución ${ }^{(15-17)}$.

\section{MARCO TEÓRICO DE LAS M\&M: UN MODELO DE CONTINUA INTERACCIÓN}

La complejidad de las M\&M es evidente al considerar las diversas teorías y modelos que subyacen en el fin académico y organizacional que configura su ejecución:

- Una teoría de la actividad. Propia del aprendizaje sociocultural, que establece las herramientas para el análisis de una interacción social compleja. Describe el aprendizaje como una colaboración entre los miembros de un equipo dinámico e inestable (cambian con el tiempo), propios de los escenarios clínicos de la práctica médica. Identifica, durante la interacción de los componentes de estos sistemas de actividad dinámicos, cuál es el eslabón responsable de su mal funcionamiento y permite un enfoque del problema ${ }^{(18)}$.

- Una teoría de aprendizaje experiencial. Establece un ciclo en el cual, inicialmente, se involucra una actividad profesional. Luego se reflexiona $y$ critica su desempeño, con una utilización posterior de esa evaluación para construir un mejor abordaje para futuros casos y una implementación sistemática del mismo cuando sea requerido, desarrollando así una maestría en la práctica profesional ${ }^{(19-20)}$. Se aprende de una experiencia o desenlace negativo, generalmente, producto de un error u omisión propia o del otro que impacta en el paciente. (Figura 1)

- Un modelo teórico de una cultura justa o de la seguridad psicológica ${ }^{(21)}$. Este postula que debe gestionarse un ambiente libre de vergüenza y de culpa (ambiente seguro), teniendo como principio que los profesionales no deberían ser culpados o castigados por los desenlaces adversos involuntarios, lo cual amenaza el proceso de aprendizaje y restauración colectiva buscado en las M\&M. Es un escenario difícil de construir dado que la culpabilidad es la preocupación principal al abordar un evento adverso. Es un aprendizaje de los casos que presentan problemas mediante revisiones que generan enseñanzas de los eventos negativos y los procesos de mejora, estableciendo un balance entre la responsabilidad individual e institucional y la seguridad del paciente con un planteamiento de retribución y restauración por el eventual daño ocasionado.

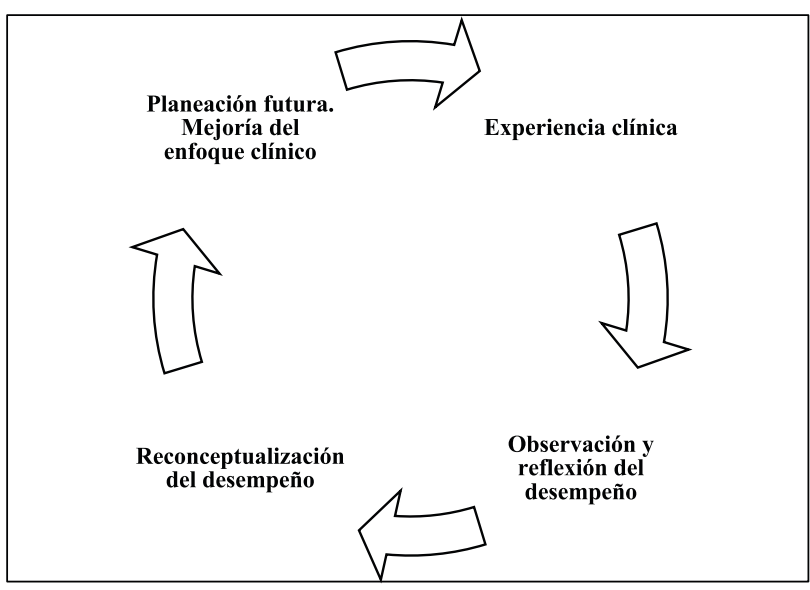

Figura 1. Ciclo de la teoría educativa, modelo teórico del aprendizaje experiencial. Fuente: adaptado de Bohnen JD et al ${ }^{(17)}$

- Un modelo teórico de seguridad (seguridad I, seguridad II) ${ }^{(22-23)}$. Se define la seguridad como "una condición donde el número de resultados adversos es lo más bajo posible" y se mide contando el número de casos en los que se falla en lugar del número de casos en los que se tiene éxito (Seguridad I). Se refiere a un modelo lineal que considera los resultados adversos como una consecuencia, donde es posible encontrar sus causas siempre que se recopile la evidencia suficiente, pudiéndose eliminar o controlar las mismas. Dado que todos los resultados adversos tienen causas susceptibles de ser descubiertas, se deduce que todos los eventos no deseados podrían ser prevenidos. Se ha establecido e incorporado por algunas organizaciones como una política de cero accidentes o de cero daños ${ }^{(23)}$. Una evolución en el modelo y su aplicación cambia la definición de seguridad anterior, de "evitar que algo salga mal" a "asegurar que todo salga bien", donde se analiza el evento adverso mediante el entendimiento de cómo los procesos similares resultan bien (modelo no lineal), 
aumentando la capacidad de tener éxito en diferentes condiciones con un número de resultados esperados y aceptables lo más alto que sea posible (seguridad II) ${ }^{(24)}$.

- Un modelo teórico de la segunda víctima. Se plantea que la incidencia y el posterior análisis de estos procesos de "error y reparación" afectan, simultáneamente, a cada uno de los actores de las M\&M y, por ende, a la institución como el lugar de práctica clínica diaria. Se considera al personal asistencial involucrado en un evento adverso como una segunda víctima, merecedor de una intervención en lo organizacional e individual. Los cambios personales derivados de esta gestión organizacional pueden modificar positivamente la operación del servicio y, a su vez, las modificaciones institucionales suscitan reglas e instrucciones que guían e impactan favorablemente en el comportamiento individual que crea ambientes seguros para los pacientes al fomentar la no repetición de eventos similares ${ }^{(24)}$.

- EI aprendizaje basado en el caso (ABC). Es la estrategia metodológica que articula los anteriores modelos teóricos al promover una discusión aroumentativa sobre un desenlace negativo e inesperado (caso clínico problema). Asimismo, permite a los participantes examinar cuidadosamente los errores en la práctica clínica para utilizarlos como oportunidades para aprender y desarrollar un ajuste en sus competencias clínicas para su implementación futura en la actividad diaria ${ }^{(18)}$. Se desarrolla un ejercicio reflexivo y un aprendizaje profundo mediante la integración de los conocimientos teóricos y prácticos al reforzar el razonamiento clínico ${ }^{(19-20)}$

La ejecución exitosa de una M\&M de acuerdo con su fin educativo, requiere de una interacción continua de estos modelos teóricos durante la misma, un clima propicio de aprendizaje y asimismo, la necesidad de minimizar las secuelas de dicha exposición pública de la falla en el proceso de atención que derivó en el evento adverso.

Por lo anterior, una M\&M se constituye en un escenario integrador de políticas de educación médica, seguridad del paciente y mejoramiento de la calidad profesional e institucional, tal como se esquematiza en la Figura 2.

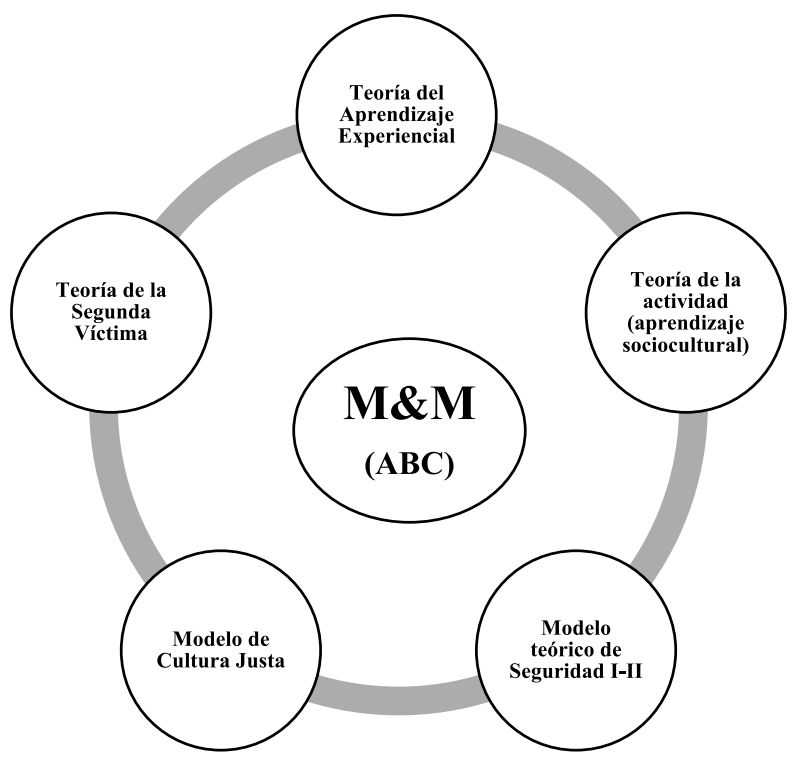

Figura 2. Modelo teórico global e interactivo de las $M \& M, A B P$ : aprendizaje basado en el caso. Se tuvo en cuenta los modelos teóricos de las M\&M. Fuente: creación propia

\section{ANÁLISIS DE LOS PROCESOS E IMPLEMENTACIÓN DE LAS M\&M}

Para su adecuado desarrollo, las M\&M deben estimar unos dominios temáticos que faciliten el análisis de los casos y la posible identificación de focos de intervención ${ }^{(25)}$ (Tabla 1).

La exposición continua de los autores durante su formación profesional y vida académica (participación continua en $M \& M$ en pregrado y posgrado) permite afirmar que, en la mayoría de las instituciones de nuestro país, no existe un libreto metodológico formalizado, ni se logra el cumplimiento de los dominios descritos con un menoscabo de la herramienta educativa $y$ de las oportunidades de mejoramiento producto de sus resultados. A la luz de la teoría experiencial del aprendizaje, no hay un cumplimiento rutinario de los procesos de reflexión, retroalimentación y construcción de estrategias formativas con medición sistemática de sus resultados de parte del estudiante y de sus docentes ${ }^{(19-20)}$. 
Tabla 1. Dominios de las M\&M

\begin{tabular}{|c|c|c|c|}
\hline Dominios & Estructura & Proceso & Contenido \\
\hline \multirow{6}{*}{$\begin{array}{l}\text { Puntos para } \\
\text { considerar } \\
\text { en los } \\
\text { dominios }\end{array}$} & $\begin{array}{l}\text { Estructura establecida con } \\
\text { claridad }\end{array}$ & $\begin{array}{l}\text { Desarrollo de casos poten- } \\
\text { cialmente significativos para } \\
\text { el aprendizaje * }\end{array}$ & Discusión académica del caso * \\
\hline & $\begin{array}{l}\text { Personal responsable de } \\
\text { presentar el caso * }\end{array}$ & Responsabilidad * & Debate educativo \\
\hline & $\begin{array}{l}\text { Participación } \\
\text { multidisciplinaria * }\end{array}$ & $\begin{array}{l}\text { Neutralidad, sin ambiente de } \\
\text { confrontación }\end{array}$ & $\begin{array}{l}\text { Eliminar la preocupación de culpa- } \\
\text { bilidad o castigo }\end{array}$ \\
\hline & Frecuencia establecida * & Compromisos del docente & Ambiente propicio de aprendizaje * \\
\hline & $\begin{array}{l}\text { Retroalimentación } \\
\text { académica }\end{array}$ & Compromisos del estudiante & $\begin{array}{l}\text { Mentalidad abierta para presentar } \\
\text { iniciativas de solución }\end{array}$ \\
\hline & & $\begin{array}{l}\text { Compromisos de la } \\
\text { institución }\end{array}$ & \\
\hline
\end{tabular}

* Dominios mayoritariamente cumplidos en nuestro país. Explicación en el texto. Fuente: adaptado de ACGME ${ }^{(3)}$

En consonancia con lo anterior, hay un vacío en la reglamentación institucional para su operación en términos de forma $y$ alcances de las reuniones, estableciéndose un posible currículo oculto en la formación profesional de los futuros médicos: las M\&M tienen códigos de comportamiento, ejecución y participación tácitos y no siempre basados en aspectos académicos que podrían incidir, desfavorablemente, en la educación médica de los asistentes ${ }^{(26-27)}$. En Colombia, existe una M\&M con metodología definida y de obligatorio cumplimiento que evalúa la calidad de la atención hospitalaria en casos de morbimortalidad materno-fetal, desde una perspectiva de la salud pública nacional y bajo una política de seguridad del paciente ${ }^{(28)}$.

Igualmente, existe un vacío normativo de las sociedades científicas en el tema, reflejando de esta manera una ausencia de interés de parte de las instituciones reguladoras de la actividad profesional médica.

Las características operativas de las M\&M varían de acuerdo con el caso a analizar, el contexto organizacional (IPS explícitamente académicas $\nabla$ s. IPS no académicas, IPS públicas $\nabla$ s. IPS privadas) y la condición del servicio: quirúrogico (cirugía general y subespecialidades, neurocirugía, ortopedia, etc.) o no quirúrogico (pediatría, medicina interna y subespecialidades, fisiatría, radiología, etc.). Del mismo modo, hay variabilidad en sus alcances y metodologías de discusión, pues examina, inclusive, los problemas de todo el sistema e indaga sobre la responsabilidad de las IPS en el caso, de acuerdo con lo estipulado en el servicio ${ }^{(29)}$.

Un formato de las M\&M con realización de fases secuenciales, producto de un abordaje integral del tema e implementado con éxito en la Universidad de Colorado (EE. UU.), se expone en la Tabla $2^{(15)}$. Su adaptación en un contexto local es factible y representa una oportunidad para responder a los cuestionamientos técnicos ya enunciados.

Cabe anotar, que el rol activo, un enfoque académico y el asertividad del docente que guía la discusión, su interacción con el estudiante o personal involucrado en el caso y, los participantes en la M\&M dentro de un ambiente de aprendizaje seguro, en ausencia de vergüenza, miedo y culpa, son determinantes en el éxito de la reunión como estrategia de aprendizaje ${ }^{(30)}$.

Entre las dificultades que inciden en su ejecución y que impactan negativamente en el cumplimiento de los objetivos educativos, está el procurar la posible resolución de múltiples problemas de forma simultánea en el caso a tratar, que pueden ser de orden institucional (logística o recursos tecnológicos), organizacional (recurso humano o políticas de funcionamiento) y del individuo (derivados de un inadecuado desempeño profesional, profesionalismo médico), o la mezcla de todos ellos ${ }^{(22)}$. 
Tabla 2. Las M\&M: eslabones secuenciales de una estrategia metodológica de $A B C$

\begin{tabular}{|c|c|c|}
\hline Etapas M\&M & Ítems & Responsables \\
\hline \multirow{6}{*}{$\begin{array}{l}\text { Preparación } \\
\text { previa }\end{array}$} & 1. Logística de la reunión & \multirow{6}{*}{$\begin{array}{l}\text { Docente involucrado. } \\
\text { Residente involucra- } \\
\text { do o designado. } \\
\text { Jefe del Servicio. }\end{array}$} \\
\hline & 2. Línea de tiempo previo al caso & \\
\hline & 3. Selección del caso & \\
\hline & 4. Preparación del caso & \\
\hline & 5. Revisión del caso y puntos de enseñanza & \\
\hline & 6. Preparación y arreglo final & \\
\hline \multirow{5}{*}{ La reunión } & 1. Introducción & \multirow{5}{*}{$\begin{array}{l}\text { Docente involucrado. } \\
\text { Residente involucra- } \\
\text { do o designado. } \\
\text { Jefe del Servicio. } \\
\text { Personal asistencial y } \\
\text { en entrenamiento. } \\
\text { Expertos invitados. }\end{array}$} \\
\hline & 2. Presentación del caso & \\
\hline & 3. Análisis del caso & \\
\hline & 4. Desarrollo de acciones futuras & \\
\hline & $\begin{array}{l}\text { 5. Cierre de la discusión } \\
\text { Materiales y métodos adicionales: agenda del caso expuesta. } \\
\text { Hoja de reflexión del residente. Herramientas de evaluación }\end{array}$ & \\
\hline \multirow{2}{*}{ Posreunión } & 1. Evaluación estructurada de la reunión & \multirow{2}{*}{$\begin{array}{l}\text { Presentador del caso. } \\
\text { Docente involucrado. } \\
\text { Auditoria médica. } \\
\text { Jefe del Servicio. }\end{array}$} \\
\hline & 2. Documentación de la M\&M & \\
\hline
\end{tabular}

Fuente: adaptado de Tad-y D, et al. (27)

Las M\&M se han enfocado en el análisis de factores derivados del desempeño de los individuos y, de forma secundaria, en los fallos institucionales. Sin embargo, es reconocido que el enfoque único al error humano no es suficiente dado que "el problema no es la gente mala; el problema es que el sistema necesita ser más seguro" (31).

En una evaluación de los posibles responsables de eventos adversos institucionales, la falta de recursos supera en gran medida a los errores humanos como un factor directamente relacionado con los desenlaces, en un $86 \%$ vs. $14 \%$ de los casos, respectivamente, sin desconocer su interacción continúa en la actividad clínica diaria ${ }^{(22)}$. Es por esto, que las $M \& M$ deben tener en consideración objetivos educativos individuales e institucionales, promoviendo el aprendizaje y la reflexión crítica de los errores, procurando un incremento en la calidad en la prestación de los servicios de salud ${ }^{(18)}$.

\section{EL PAPEL DE LAS M\&M EN LA EDUCACIÓN MÉDICA}

La generación de un clima de aprendizaje favorable en la operación de una $M \& M$, redunda en un posible cambio de conductas en los individuos y las instituciones con un impacto positivo en la salud de los pacientes ${ }^{(32)}$. Esto contempla la identificación de resultados adversos adyacentes dentro del caso, una discusión abierta de los errores y la difusión del aprendizaje, como parte de una política institucional que promueva estrategias asociadas con el éxito individual y organizacional y teniendo en cuenta que la ocurrencia de los resultados negativos es un fenómeno multicausal no lineal ${ }^{(12,18,22)}$.

Adicionalmente, en su implementación se debe evaluar y promover el cumplimiento de las seis competencias centrales, esperadas en la formación de los estudiantes en los diferentes programas educativos (Core Competences), con el fin de favorecer su desarrollo en los futuros profesionales ${ }^{(33-54)}$. Esto se ejemplifica en un caso clínico de un evento adverso, como la infección del sitio operatorio (ISO) tras la instalación de un catéter central: se espera la elaboración y desarrollo de conceptos como la importancia de la asepsia y antisepsia, la técnica quirúrơica, los cuidados posteriores al procedimiento y los conocimientos de una ISO. Esta M\&M debe abordar, además, puntos clave de la seguridad del paciente que impacten 
en la calidad institucional $y$, de igual manera, procurar el reforzamiento proactivo de la necesidad del trabajo bien hecho, fortaleciendo las oportunidades de éxito ${ }^{(22)}$ (Tabla 3).

Tabla 3. Evaluación de las seis competencias centrales (Core Competences) en la formación de residentes. Caso hipotético de una M\&M en un paciente con ISO tras la inserción de un catéter central

\begin{tabular}{|c|c|c|c|c|c|}
\hline \multicolumn{6}{|c|}{ Competencias centrales en la educación médica procuradas en una M\&M } \\
\hline $\begin{array}{l}\text { Cuidado del } \\
\text { paciente }\end{array}$ & $\begin{array}{l}\text { Conocimiento } \\
\text { médico }\end{array}$ & $\begin{array}{l}\text { Aprendizaje } \\
\text { basado en la } \\
\text { práctica }\end{array}$ & $\begin{array}{l}\text { Habilidades de } \\
\text { comunicación }\end{array}$ & Profesionalismo & $\begin{array}{l}\text { Sistema basado } \\
\text { en la práctica }\end{array}$ \\
\hline $\begin{array}{l}\text { Discutir la } \\
\text { importancia } \\
\text { de proteger al } \\
\text { paciente de las } \\
\text { infecciones del } \\
\text { sitio operatorio. }\end{array}$ & $\begin{array}{l}\text { Aprendizaje de } \\
\text { las infecciones } \\
\text { del sitio } \\
\text { operatorio. }\end{array}$ & $\begin{array}{l}\text { Promover el } \\
\text { adecuado } \\
\text { manejo e } \\
\text { instalación de } \\
\text { los catéteres } \\
\text { centrales } \\
\text { para evitar las } \\
\text { infecciones. }\end{array}$ & $\begin{array}{l}\text { Comunicar y enseñar } \\
\text { el cumplimiento } \\
\text { de los procesos, } \\
\text { al personal de } \\
\text { enfermería } \\
\text { encargado de la } \\
\text { manipulación de los } \\
\text { catéteres centrales. }\end{array}$ & $\begin{array}{l}\text { Honestidad en } \\
\text { reconocer las posibles } \\
\text { omisiones o acciones } \\
\text { inseguras en la técnica } \\
\text { o en los cuidados } \\
\text { postoperatorios. } \\
\text { Actualización en ISO y } \\
\text { sepsis por catéter. }\end{array}$ & $\begin{array}{l}\text { Implementación } \\
\text { institucional } \\
\text { de políticas de } \\
\text { adecuado manejo } \\
\text { de catéteres } \\
\text { centrales. }\end{array}$ \\
\hline
\end{tabular}

Fuente: adaptado de ACGME $^{(3)}$

Igualmente, se deben fomentar las discusiones multidisciplinarias donde se argumente sobre la evidencia y se fomenten habilidades de comunicación, profesionalismo y trabajo en equipo, así como el establecimiento de una cultura de seguridad que mejore la atención del paciente y sus resultados.

Realizar una adecuada selección de los casos a estudiar es un reto procedimental dentro de una $M \& M$, debido a la necesidad de priorizar de acuerdo con el contexto local administrativo, las necesidades docentes, la gravedad y la relevancia del caso. Esto incluye identificar eventos adversos y problemas de seguridad mediante varias fuentes de datos como son el registro de complicaciones, los sistemas de reporte de incidentes, encuestas y llamadas telefónicas a los pacientes, así como la información de proveedores, entre otras. Esta recolección de datos requiere de un análisis integral para escoger adecuadamente los casos para el estudio (esto se ha convertido en un verdadero desafío), ya que llega a documentarse en algunas instituciones hasta 5.000 reportes de casos susceptibles de análisis cada año ${ }^{(22)}$. Una propuesta categorización, de casos potencialmente candidatos a ser escogidos sería: a. Casos tradicionales de $\mathbf{M} \& \mathbf{M}$ : resultados no esperados, diagnóstico inoportuno, ausencia de él, etc. Identificados por el jefe de los residentes, médicos o coordinador del servicio.

b. Casos de M\&M enfocados en los sistemas: casos comunes, pero con error en el complejo sistema de atención del paciente. El diagnóstico final no es tan importante. La identificación se hace por evaIuación de pares, revisión de incidentes y mortalidad, jefe de residentes, médicos del servicio y auto reportes ${ }^{(15)}$.

La decisión final en la escogencia, dependerá de la institución, el servicio, los intereses de los actores y del impacto del caso en la comunidad hospitalaria $y$ académica, entre otros factores ${ }^{(22,29)}$.

Durante el desarrollo de las M\&M es importante registrar los puntos de alto aprendizaje (presentación del caso, discusión argumentativa y reflexiones finales) y las oportunidades de mejoramiento, enfatizando en el seguimiento y propuesta de cambios para evitar la recurrencia de los problemas ${ }^{(29)}$.

La evaluación del aprendizaje se ha abordado mediante la búsqueda de un adecuado cumplimiento de los objetivos planteados al inicio de la reunión, 
interrogando además si el aprendizaje desarrollado anticipa los cambios en la práctica clínica (aprendizaje profundo) ${ }^{(33)}$.

Una evaluación del impacto de la discusión del caso en la conducta futura de los médicos, mediante encuestas reflexivas, registró una asociación entre la percepción del profesional sobre la relevancia del caso y el proceso de reflexión sobre ella, concluyendo que mientras más profundo se logre el aprendizaje más probable será el cambio en el comportamiento ${ }^{(15,19)}$.

EI logro de los objetivos educativos tras una M\&M es un asunto poco explorado. Abordajes previos del tema han establecido unas percepciones de bajos indicadores de cambio en la práctica clínica de los profesionales involucrados en ellas, así como de las expectativas de mejoría ${ }^{(35)}$. Lo anterior pareciera estar relacionado con las variaciones en la ejecución de las M\&M en las escogencia de los casos, ambientes de aprendizaje, discusión asertiva y académica, entre otros, $y$ el desconocimiento del papel individual de cada uno de estos factores ${ }^{(35)}$, así como sus interacciones de acuerdo con el modelo teórico integrado, esquematizado en la figura 2.
Es posible que aspectos como la información completa y clara, el desarrollo de un clima de aprendizaje favorable con retroalimentación y discusiones respetuosas, la motivación de los asistentes, el significado de la urgencia del caso y la realización de las reuniones con un objetivo claro y alcanzable, propicien un empoderamiento enfocado en el cambio que, sumado al seguimiento y mantenimiento de ellas, podrían servir como "mediadores potenciales para impulsar el aprendizaje", según lo argumentado por DeVos y cols., en sus investigaciones ${ }^{(22,36)}$.

Lo anterior, hace manifiesto un vacío en la evaluación de la calidad de las M\&M y las variables relacionadas con su éxito como escenario académico, en razón de la poca información disponible con respecto al impacto esperado en la educación médica y en el mejoramiento de la calidad, que son el principio base del desarrollo de estas reuniones ${ }^{(32.35)}$. No obstante, se han podido identificar factores a nivel individual y colectivo que se comportan como facilitadores para el éxito de las M\&M y deben ser tenidos en cuenta en su ejecución ${ }^{(36)}$ (Tabla 4).

Tabla 4. Recomendaciones para la práctica exitosa de las M\&M

\begin{tabular}{|c|c|}
\hline Recomendación & Detalles \\
\hline Urgencia & $\begin{array}{l}\text { Temas relevantes para el público, que sean aplicados en la práctica diaria y apoyados por datos de } \\
\text { incidencia. }\end{array}$ \\
\hline Información & Información clara apoyada en la literatura. \\
\hline Planificación & Determinar los actores de la M\&M, detallando un plan de acción, seguimiento y evaluación. \\
\hline Motivación & Discusión interactiva y con retroalimentación. \\
\hline Anticipación & Anticipación de las acciones y planeación para contrarrestar problemas. \\
\hline Entrada & $\begin{array}{l}\text { Asegurar la presencia y apoyo de todo el personal, mediante la participación del personal con expe- } \\
\text { riencia o de otras disciplinas. }\end{array}$ \\
\hline Receptividad & Mantener una mentalidad abierta que acepte aportes de todos los involucrados. \\
\hline Ajuste & Reuniones de las subespecialidades y discusiones multidisciplinarias. \\
\hline Recursos & $\begin{array}{l}\text { Tiempo protegido para el desarrollo de las } M \& M \text {, se debe considerar destinar un tiempo del personal } \\
\text { asistencial para la participación en la reunión. }\end{array}$ \\
\hline Datos & $\begin{array}{l}\text { Soportarse en datos locales y regionales que permitan monitorear y evaluar el desempeño particular, } \\
\text { además, de realizar una evaluación comparativa en los planes de mejora. }\end{array}$ \\
\hline
\end{tabular}

Fuente: adaptado de Vos, M.S. ${ }^{(21)}$ 


\section{REFLEXIONES FINALES}

Las M\&M, en consonancia con sus objetivos organizacionales y académicos, direccionados al mejoramiento de la calidad de la atención hospitalaria y la seguridad del paciente, deben operar con una metodología basada en el $A B C$ que integre los diversos modelos teóricos expuestos en el presente escrito. Su implementación metodológica, en pro de estos propósitos, debe ser sistemática y disciplinada, equilibrando las cuestiones administrativas o de otra índole no académica durante su ejecución.

Asimismo, su actividad debe motivar cambios individuales y organizacionales, mediante la educación a todo el personal de salud involucrado en el cuidado del paciente, así como la participación activa de las instituciones con su normatividad y cumplimiento.

La evaluación de los resultados de aprendizaje en el pregrado, posogrado y en la educación médica continuada derivados de las M\&M es un tema aún poco estudiado, así como el posible impacto educativo diferencial de acuerdo al nivel formativo del personal expuesto. Por consiguiente, se hace necesario una metodología de evaluación por objetivos y un reconocimiento de su papel pedagógico en todos los niveles, incluyendo la educación médica continuada.

El papel de las M\&M como herramienta educativa, tal y como quedó expuesto en el presente documento, no está totalmente desarrollado. Se ha considerado, mayoritariamente, como un instrumento evaluativo de la calidad de la atención y la seguridad del paciente, siendo un desafío su implementación y fortalecimiento como fuente de cambio en las instituciones y los individuos, producto de un aprendizaje profundo, constructivo y reflexivo.

Finalmente, se hace evidente un campo para futuras investigaciones en el tema de la educación médica como instrumento de impacto organizacional $y$ administrativo, con las M\&M como un foco de intervención determinante en el ejercicio médico actual $y$ futuro.

\section{CONFLICTOS DE INTERESES}

Ninguno por declarar. Todos los autores participaron en las diferentes fases de la elaboración del presente trabajo, no se recibieron fondos de alguna entidad estatal o privada, este responde al esfuerzo e interés académico de sus autores.

\section{REFERENCIAS BIBLIOGRÁFICAS}

1. McDonnell C, Laxer R, Roy W. Redesigning a Morbidity and Mortality Program in a University-Affiliated Pediatric Anesthesia Department. Jt Comm J Qual Patient Saf. 2010;36(3):117-25. DOI 10.1016/S1553$7250(10) 36020-X$

2. Aeby TC. The role of morbidity and mortality (M\&M) conferences in medical education. Hawaii Med J. 2011;70(2):39-40.

3. ACGME. Common Program Requirements [internet]. [Consultado 2019 ago 01]. Disponible en: https://cutt. Iy/Re3MHaP

4. Berenholtz S, Hartsell T, Pronovost P. Learning From Defects to Enhance Morbidity and Mortality Conferences. Am J Med Qual. 2009;24(3):192-5. DOI 10.1177/1062860609332370.

5. Aboumatar H, Blackledge C, Dickson C, Heitmiller E, Freischlag J, Pronovost P. A Descriptive Study of Morbidity and Mortality Conferences and Their Conformity to Medical Incident Analysis Models: Results of the Morbidity and Mortality Conference Improvement Study, Phase 1. Am J Med Qual. 2007;22(4):232238. DOI $10.1177 / 1062860607303292$.

6. Colombia. Ministerio de Protección Social. Lineamientos para la implementación de la Política de Seguridad del Paciente [internet]. [Consultado 2019 ago 1]. Disponible en: https://cutt.ly/9rwyIsk

7. OMS [internet]. Ginebra: OMS; 1998. [Consultado 2019 ago 1]. Disponible en: https://cutt.ly/Se31n85

8. SIN-NL [Internet]. Irlanda: SIN-NL; 2006. [Consultado 2019 ago 03]. Disponible en: https://cutt.ly/Ke31XtA

9. Gordon LA. Can Cedars-Sinai's M+M Matriz save surgical education? Bull Am Coll Surg. 2004;89(6):16-20.

10. López Herrera Ju, Gómez López J. Recomendaciones para la implementación de herramientas en programas de atención segura en instituciones prestadoras de salud (IPS). Arch. med. (Col). 2011;11(2):169-82.

11. España. Ministerio de Sanidad y Política Social. Estudio IBEAS: prevalencia de Efectos Adversos en 
hospitales de Latinoamérica [internet]. [Consultado 2019 ago 04]. Disponible en: https://cutt.ly/6rRTUAE

12. Orlander JD, Barber TW, Fincke BG. The morbidity and mortality conference: the delicate nature of learning from error. Acad Med. 2002;77(10):1001-6. DOI 10.1097/00001888-200210000-00011.

13. Castro A. Necesidad de evaluar los programas de formación de cirugía en Colombia. Rev Colomb Cir. 2016:31:161-4.

14. Sakowska M, Connor S. The value of voluntary morbidity and mortality meetings at a New Zealand metropolitan hospital. N Z Med J. 2008;121(1279):57-65.

15. Tad-y D, WaId H. Systems and Quality M\&M Toolkit [internet]. [Consultado 2019 ago 6]. Disponible en: https://cutt.ly/jrRTOuS

16. Bal G, Sellier E, Tchouda SD, François P. Improving quality of care and patient safety through morbidity and mortality conferences. J Healthc Qual. 2014;36(1):2936. DOI 10.1111/j.1945-1474.2011.00203.x.

17. Bohnen JD, Chang DC, Lillemoe KD. Reconceiving the morbidity and mortality conference in an era of big data. An "unexpected" outcomes approach. Ann Surg. 2016;263(5):857-9. DOI 10.1097/ SLA. 0000000000001508.

18. Varpio L, Hall P, Lingard L, Schryer C. Interprofessional communication and medical error: a reframing of research questions and approaches. Acad Med. 2008;83:S76S81. DOI 10.1097/ ACM.0b013e318183e67b.

19. Gregory A, Taylor D. Morbidity and mortality conference: its purpose reclaimed and grounded in theory. Teach Learn Med. S2016;28(4):439-47. DOI 10.1080/10401334.2016.1189335.

20. McLean SF. Case-Based Learning and its Application in Medical and Health-Care Fields: A Review of Worldwide Literature. J Med Educ Curric Dev. 2016;22(3). DOI 10.4137/JMECD.S20377.

21. Khatri N, Brown GD, Hicks L. From a blame culture to a just culture in health care. Health Care Manage Rev. 2009;34(4),312-22. DOI 10.1097/ HMR.0b013e3181a3b709.

22. Vos M. Healthcare improvement based on learning from adverse outcomes. [Tesis doctoral] Leiden: Medicine / Leiden University Medical Center; 2018.
[Consultado en 2019 agosto 06]. Disponible en: https:// openaccess.leidenuniv.nI/handle/1887/67419

23. Hollnagel E, Wears RL, Braithwaite J. From Safety-I to Safety-II: A White Paper [internet]. [Consultado 2019 ago 07]. Disponible en: https://cutt.ly/ZrRYqVH

24. Scott SD, Hirschinger LE, Cox KR, McCoig M, Brandt J, Hall LW. The natural history of recovery for the healthcare provider "second victim" after adverse patient events. BMJ Quality \& Safety. 2009;18(5):32530. DOI 10.1136/qshc.2009.032870.

25. Flynn-O'Brien KT, Mandell SP, Eaton EV, Schleyer AM, McIntyre LK. Surgery and Medicine Residents' Perspectives of Morbidity and Mortality Conference: An Interdisciplinary Approach to Improve ACGME Core Competency Compliance. J Surog Educ. 2015;72(6):e258-66. DOI 10.1016/j.jsuro. 2015.05.015.

26. Hill E, Bowman K, Stalmeijer R, Hart J. You've got to know the rules to play the game: how medical students negotiate the hidden curriculum of surgical careers. Med Educ. 2014;48(9):884-94. DOI 10.1111/ medu. 12488.

27. Hafler JP, Ownby AR, Thompson BM, Fasser CE, Grigsby KD, Haidet P et al. Decoding the learning environment of medical education: a hidden curriculum perspective for faculty development. Acad Med. 2011;86(4):440-4. DOI 10.1097/ ACM.0b013e31820df8e2.

28. OPS. Colombia. Sistema de Vigilancia Epidemiológica de Mortalidad Materna Basada en la Web (SVEMMBW) [internet]. [Consultado 2019 ago 07]. Disponible en: https://cutt.1y/5rRU4zc

29. Epstein NE. Morbidity and mortality conferences: Their educational role and why we should be there. Surg Neurol Int. 2012;3(SuppI 5):S377-S88. DOI 10.4103/2152-7806.103872.

30. Smaggus A, Mrkobrada M, Marson A, Andrew Appleton. Effects of efforts to optimise morbidity and mortality rounds to serve contemporary quality improvement and educational goals: a systematic review. BMJ Qual Saf. 2018;27:74-84. DOI 10.1136/ bmjqs-2017-006632.

31. Hendee WR. To Err is Human: Building a Safer Health System. JVIR. 2001;12(1):112-3. DOI 10.1016/S10510443(01)70072-3.

32. Bechtold M, Scott S, Dellsperger K, Hall L, Nelson $\mathrm{K}$, Cox K. Educational quality improvement report: 
outcomes from a revised morbidity and mortality format that emphasised patient safety. BMJ. 2008;84(990):211-6. DOI 10.1136/qshc.2006.021139.

33. Kauffmann RM, Landman MP, Shelton J, Dmochowski RR, Bledsoe SH, Hickson GB et al. The use of a multidisciplinary morbidity and mortality conference to incorporate ACGME general competencies. J Surg Educ. 2011;68(4):303-8. DOI 10.1016/j. jsurg.2011.02.002.

34. Kravet SJ, Howell E, Wright SM. Morbidity and mortality conference, grand rounds, and the ACGME's core competencies. J Gen Intern Med. 2006;21(11):1192-4. DOI 10.1111/j.1525-1497.2006.00523.x.

35. De Vos MS, Hamming JF, Marang-van de Mheen PJ. Learning From Morbidity and Mortality Conferences: Focus and Sustainability of Lessons for Patient Care. J Patient Saf. 2017. DOI 10.1097/PTS.0000000000000440.

36. De Vos M, Hamming J, Marang-van de Mheen P. Barriers and facilitators to learn and improve through morbidity and mortality conferences: a qualitative study. BMJ Open. 2017;7(11):e018833. DOI 002010.1136/bmjopen-2017-018833. 